\title{
Syndromic management of sexually transmitted diseases at primary care level, Mozambique
}

\section{F S Mbofana, F J Brito, A Saifodine, J L Cliff}

n 1985, the World Health Organization (WHO) developed simplified treatment guidelines for STD treatment at primary healthcare level. ${ }^{1}$ After several revisions of the guidelines, WHO now recommends purely syndromic management. ${ }^{2}$

Since 1988, the Mozambican Ministry of Health $(\mathrm{MOH})$ has progressively introduced sexually transmitted disease (STD) clinics at public primary level facilities (health posts and health centres). Health workers have been trained to use simplified treatment guidelines that include syndromic management. This one-off study aimed to assess the quality of STD management and the effectiveness of referral to the STD clinic at selected sites.

\section{METHODS}

We carried out the study in 26 primary care facilities in Maputo and Quelimane cities and Manica and Tete provinces in 1995-6. Either all or randomly selected facilities were chosen. All STD patients presenting to the general adult or STD clinic were observed for one or two consecutive days. STD clinic frequency varied from daily to weekly.

We used a modified version of the WHO recommended protocol for observing STD case management. ${ }^{3}$ Denominators varied, as the whole process could rarely be observed in the same patient, because of referral to the laboratory, mother and child health services, and the STD clinic.

Where possible, we collected retrospective information in each general clinic on 100 consecutive patients who were referred to the STD clinic. We considered the patient as lost during referral if their name did not appear in the STD clinic register within the next 15 days.

\section{RESULTS}

We observed 408 patients-267 (65\%) in STD and 141 (35\%) in general clinics-at different stages of the consultation; 32 of the 42 health workers ( 38 males and four females) observed had received training in STD management guidelines; 286
(70\%) patients were women $(71 \%$ and $69 \%$ in the STD and general clinic, respectively). Table 1 shows the presenting symptoms.

Only 54/206 (26\%) eligible women were examined, compared to $64 / 85$ $(75 \%)$ eligible men $(\mathrm{p}<0.0001)$. Laboratory tests were requested in 153/206 $(74 \%)$ women and 38/85 (45\%) men $(p=0.0002)$. Syndromic diagnoses were more frequent in women $(57 \%$ against $38 \%, \mathrm{p}=0.008$ ) (table 1 ).

Rapid plasmin reagin syphilis serology that was not recommended in the guidelines was requested in 147/291 (51\%) patients. Physical and/or laboratory examinations were performed in only 33 of the 126 patients diagnosed aetiologically.

The STD and adult general clinic did not differ significantly in the proportion examined, laboratory tests requested, diagnosis, and treatment.

Only 38/197 (19\%) and 118/197 (60\%) women were advised on condom use and contact treatment respectively, compared to $59 / 68(87 \%)$ and $62 / 68(91 \%)$ men $(\mathrm{p}<0.001 ; \mathrm{p}=0.04) ; 91 / 193 \quad(47 \%)$ and $156 / 193(81 \%)$ patients were advised on condom use and contact treatment, respectively, in the STD clinic compared to $6 / 72(8 \%)$ and $34 / 72(47 \%)$ in the general clinic $(\mathrm{p}<0.001 ; \mathrm{p}<0.001)$.

Of 1193 STD patients seen in the general clinic, 54\% were referred to the STD clinic. Of those, only $41 \%$ presented at the STD clinic.

\section{DISCUSSION}

Introduction of STD clinics and simplified treatment guidelines and many years of training and supervision have resulted in a functioning STD service at

Table 1 Symptoms and diagnoses in STD patients, primary care level, Mozambique

\begin{tabular}{lclc}
\hline Female (n=254) & No $(\%)$ & Male $(\mathrm{n}=99)$ & No $(\%)$ \\
\hline Presenting symptoms & & & \\
Vaginal discharge & $193(76)$ & Urethral discharge & $46(46)$ \\
Lower abdominal pain & $130(51)$ & Genital ulcer & $41(41)$ \\
Burning urine & $51(20)$ & Burning urine & $38(38)$ \\
Genital itching & $41(16)$ & Genital itching & $27(27)$ \\
Genital ulcer & $38(15)$ & Genital vesicles & $10(10)$ \\
Genital vesicles & $10(4)$ & Bubo & $8(8)$ \\
Genital warts & $5(2)$ & Genital warts & $2(2)$ \\
\hline Female (n=197) & No (\%) & Male (n=68) & No (\%) \\
\hline Diagnoses & & & \\
Syndromic: & & Syndromic: & \\
Vaginal discharge & $102(52)$ & Genital ulcer & $14(21)$ \\
Genital ulcer & $10(5)$ & Urethral discharge & $12(18)$ \\
Total & $112(57)$ & Aetiological: & $26(38)$ \\
Aetiological: & & Gonorrhoea & \\
Syphilis & $26(13)$ & Syphilis & $13(19)$ \\
Gonorrhoea & $20(10)$ & Non-gonococcal urethritis & $11(16)$ \\
Trichomonas & $18(9)$ & Lymphogranuloma venereum & $6(10)$ \\
Non-gonococcal urethritis & $10(5)$ & Herpes & $3(4)$ \\
Lymphogranuloma venereum & $4(2)$ & Chancroid & $2(3)$ \\
Candidiasis & $4(2)$ & & \\
Chancroid & $3(2)$ & & $42(62)$ \\
Total & $85(43)$ & & \\
\hline
\end{tabular}


the primary care level. The high proportion $(70 \%)$ of women patients seen confirms that primary level STD services tend to treat more women than men, in contrast with secondary level STD clinics. $^{4}$

The STD clinic performed better than the general clinic in health education. This advantage was offset by large patient losses during referral to the STD clinic. Adherence to guidelines for laboratory use was poor, and referral to the laboratory probably contributed to patient losses.

Rapid and effective treatment of patients with STDs using syndromic management offers a means to interrupt transmission of HIV and other STDs. ${ }^{5}$ A proposal by the $\mathrm{MOH}$ to integrate treatment in the general clinic and introduce purely syndromic management should remedy some of the deficiencies found in this study.

\section{CONTRIBUTORS}

All authors participated in the study design, fieldwork, data analysis, and writing.

Sex Transm Inf 2002;0:e2

www.sextransinf.com/cgi/content/full/78/1/e2

\section{Authors a.........}

Authors' affiliations

F S Mbofana, F J Brito, A Saifodine, J L Cliff,

Faculdade de Medicina, Universidade Eduardo

Mondlane, Maputo, Mozambique

Correspondence to: Dr Julie Cliff, Faculdade de Medicina, Universidade Eduardo Mondlane, CP 257, Maputo, Mozambique;

jcliff@tropical.co.mz

Accepted for publication 25 October 2001

\section{REFERENCES}

1 World Health Organization. Simplified approaches for sexually transmitted disease
(STD) control at the primary health care (PHC) level. WHO/VDT/85.347. Geneva: WHO, 1985.

2 World Health Organization. Management of sexually transmitted diseases. WHO/GPA/TEM/94.1 Rev 1. Geneva: WHO, 1997.

3 World Health Organization. Protocol for the assessment of STD case management through health facility survey. Geneva: WHO, 1993.

4 Dos Santos RB, Folgosa EM, Fransen, L. Reproductive tract infections in Mozambique: case study of integrated services. In: Germain A et al, eds. Reproductive tract infections. Global impact and priorities for women's reproductive health. New York: Plenum Press, 1992:343-59.

5 Fleming DT, Wasserheit JN. From epidemiological synergy to public health policy and practice: the contribution of other sexually transmitted diseases to sexual

transmission of HIV infection. Sex Transm Inf 1999;75:3-17. 